\title{
Identificación de variedades de maíz y prácticas agrícolas en el valle de Hualfín (Catamarca, Argentina)
}

\author{
Celeste Valencia, Marina Flores, Federico Wynveldt y Bárbara Balesta \\ Laboratorio de Análisis Cerámico, Facultad de Ciencias Naturales y Museo \\ Universidad Nacional de La Plata \\ celes_valencia@hotmail.com marin481@hotmail.com \\ fwynveldt@yahoo.com.ar barbarabalesta@hotmail.com
}

Recibido: $10 / 11 / 2015$

Aceptado: 10/6/2016

\begin{abstract}
RESUMEN
Este artículo se centra en las excavaciones realizadas en Cerro Colorado de La Ciénaga (valle de Hualfín, Catamarca, Argentina) y en la descripción de las evidencias de prácticas agrícolas asociadas a él. Se trata de un sitio de altura, que comprende más de 100 recintos y que ha sido adjudicado a momentos tardíos de la historia prehispánica, siglos XIII a XVI. Las excavaciones se realizaron en el Recinto 35 y se obtuvieron restos de maíces correspondientes a espigas enteras con y sin granos, termoalteradas, que han posibilitado -por primera vez en el valle- la identificación de ocho variedades que incluyen maíces duros, semiduros y dulces. El contexto de hallazgo, que incluye pozos circulares con sedimento fino, vasijas finas y ordinarias y restos arqueofaunísticos y líticos, nos lleva a postular que en este espacio se procesaron comidas y bebidas y se manufacturaron artefactos líticos.
\end{abstract}

Palabras clave: maíz, prácticas agrícolas, noroeste argentino, período Tardío.

\section{Identification of Maize Varieties and Agricultural Practices in Hualfin Valley (Catamarca, Argentina)}

\begin{abstract}
This paper focuses on excavations carried out in Cerro Colorado de La Ciénaga (Catamarca, Argentina) and the description of associated evidences of agricultural practices. The site, located on top of a hill, comprises more than 100 rooms and was inhabited from $13^{\text {th }}$ to $16^{\text {th }}$ centuries. Excavations were carried out in Dwelling 35 and recovered numerous carbonized maize remains -with, and without grains-; eight maize varieties are identified for the first time in the valley, belonging to hard, semi-hard and sweet species. The context includes circular pits containing smooth sediment, fine and coarse pottery fragments, and faunal and lithic remains. The evidences suggest that the space was used to produce meals and beverages and to manufacture lithic artefacts.
\end{abstract}

Key words: maize, agricultural practices, Argentinean Northwest, Late Period.

Sumario: 1. Introducción. 2. La presencia de maíz en sitios arqueológicos del Noroeste argentino. 3. Evidencias indirectas de explotación agrícola en el valle de Hualfín. 4. Las excavaciones en el Recinto 35. 5. Los hallazgos en el Recinto 35. 6. Los maíces. 7. Discusión. 8. Referencias bibliográficas.

\section{Introducción}

Los recursos vegetales han tenido un rol protagónico en el desarrollo de las sociedades humanas. El maíz es uno de los recursos más relevantes; su gran adaptabilidad y plasticidad han constituido ventajas significativas, evidenciadas por el hecho de que, si bien su origen es americano, hoy en día representa la segunda planta alimenticia con distribución a nivel mundial (Staller 2010). Entre los pueblos americanos, el maíz adquirió un lugar preponderante en las esferas económica, política y simbólica, 
como lo atestiguan los registros históricos y arqueológicos, así como la preeminencia que conserva actualmente en la economía y en la vida de las poblaciones de los Andes (Hastorf y Johanessen 1993; Hayashida 2008; Jennings 2005).

La mayor parte de la información que da cuenta del rol del maíz en las economías antiguas corresponde a fuentes etnohistóricas. En general estos documentos fueron escritos con propósitos administrativos y muchos de ellos refieren reclamaciones territoriales que a menudo involucraban áreas en las que se cultivaban variedades muy valoradas de maíz y/o en las que el cultivo de maíz era relevante para las economías locales. También registran datos sobre cómo era producido y consumido el maíz por las culturas nativas y revelan detalles acerca de cómo se organizaba la siembra, cosecha, almacenamiento y distribución del producto.

Asimismo, en varios de estos textos se comenta que existían distintas categorías de productos cultivados, entre los cuales los tubérculos y la quínoa se consideraban más populares, mientras que el maíz se asociaba a las elites (Garcilaso de la Vega 1800). Uno de los productos más prestigiosos que se manufacturaba con sus granos era la chicha; se han publicado ilustraciones y descripciones de Guaman Poma de Ayala (1987 [1615]) que muestran las actividades agrícolas y festividades durante las cuales se ofrendaba y consumía chicha, ofrecida por mujeres a hombres. A través de estas ilustraciones se pueden observar roles de género ya que presentan mujeres trabajando la tierra mientras los hombres roturan; las mujeres se muestran asociadas con vasijas que presumiblemente contenían chicha.

En el presente trabajo reseñamos un hallazgo que consideramos de gran importancia: los restos de maíz recuperados en las excavaciones realizadas en uno de los recintos de Cerro Colorado de La Ciénaga, un sitio tardío prehispánico del valle de Hualfín, en el noroeste argentino. Su principal objetivo es describir e identificar las razas de maíz presentes en esos restos. Por otra parte, la variabilidad observada y el contexto de recuperación nos llevan a examinar una multiplicidad de cuestiones relacionadas con el manejo del recurso en sí mismo, el conocimiento de las propiedades de las distintas razas o variedades de maíz, las prácticas agrícolas desarrolladas en la zona, las actividades de procesamiento, las áreas de actividad y elementos asociados al procesamiento, los ejecutores de las tareas, y las personas que podrían haber consumido el recurso.

El valle de Hualfín se extiende desde Los Nacimientos hasta la denominada Puerta de Belén y está limitado al sur y sureste por la Sierra de Belén, al noreste por las estribaciones de las Sierras de Hualfín y Las Cuevas y al norte y noroeste por extremo sur del bloque de la Puna y la sierra de Altohuasi $(4.000 \mathrm{~m})$; por el oeste, el Cordón del Durazno, y más al poniente, la Sierra de Fiambalá, lo separan del valle de Abaucán (Figura 1). El valle abarca una superficie aproximada de $2.000 \mathrm{~km}^{2}$, con un diseño entrelazado de régimen fluvial semipermanente, siendo recorrido por el río Hualfín o Belén, que nace en la confluencia de los ríos Nacimientos y Durazno, y desde allí emprende un recorrido con rumbo noreste-sudoeste, durante el cual recibe el aporte de diversos tributarios tales como Loconte, Villavil, Corral Quemado y del Agua Clara, entre otros. Desde el punto de vista geológico el área corresponde a las Sierras Pampeanas Noroccidentales (Caminos 1979) y desde el punto de vista arqueológico a la Región Valliserrana (González y Pérez 1972). 
Figura 1: Mapa del valle de Hualfín, con indicación de sitios arqueológicos.

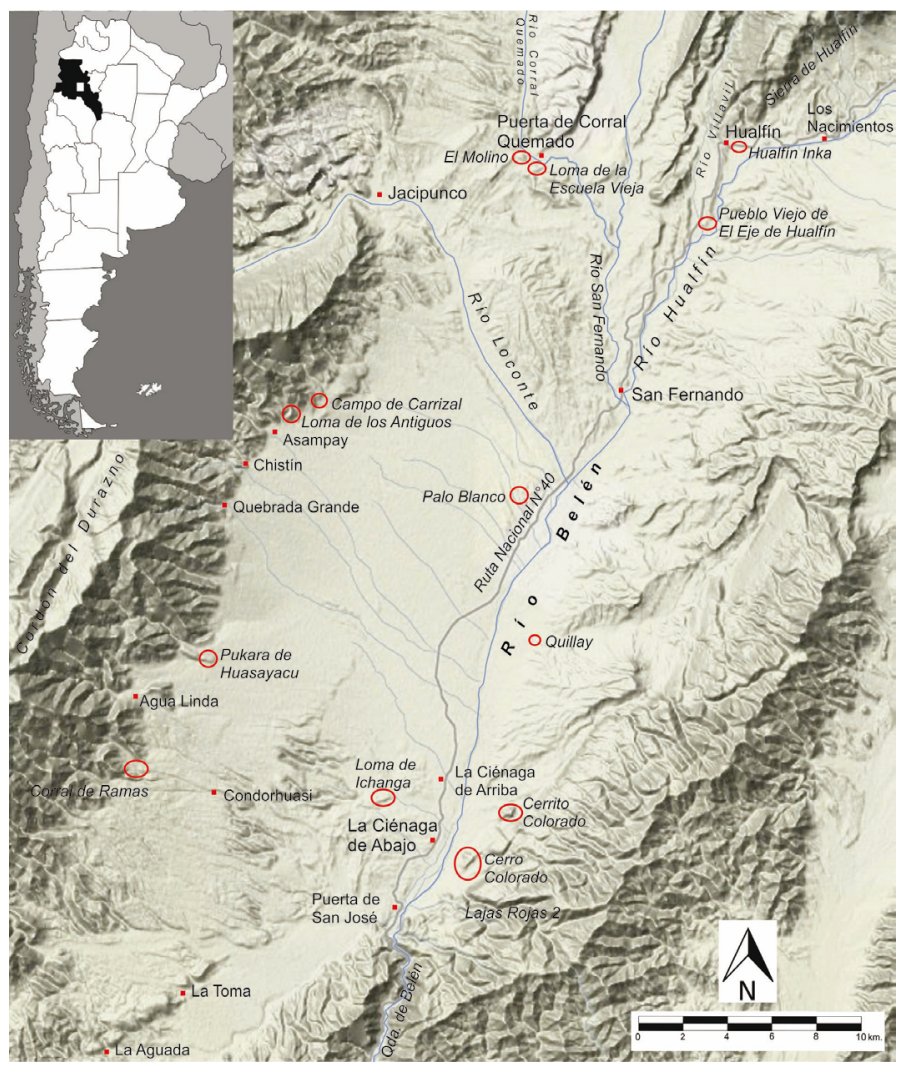

La localidad de La Ciénaga se ubica en el sector sur del valle de Hualfín, sobre la Ruta Nacional $\mathrm{N}^{\circ} 40$, entre 4 y $8 \mathrm{~km}$ al norte de la quebrada de Belén; en ella se han registrado numerosos sitios arqueológicos adscritos al período $\operatorname{Tardío}^{1}$; muchos de ellos corresponden a lo que se ha denominado como pukaras o sitios en altura. Uno de los sitios en altura más relevantes es el Cerro Colorado de La Ciénaga; se localiza en las coordenadas $27^{\circ} 31^{\prime} 38,8^{\prime \prime} \mathrm{S}$ y $66^{\circ} 58^{\prime} 14,6^{\prime \prime} \mathrm{O}$ y se eleva $150 \mathrm{~m}$ sobre el terreno circundante, sobre una lomada en la orilla oriental del río Hualfín.

El sitio arqueológico fue registrado por primera vez a principios del siglo XX (Bruch 1911); no obstante, las primeras excavaciones se llevaron a cabo en 1981 (Sempé y Pérez 1988), en el denominado Recinto 48, ubicado en el Sector Central. Posteriormente, el equipo del Laboratorio de Análisis Cerámico (LAC) -Facultad de Ciencias Naturales y Museo de la UNLP- realizó excavaciones en el Recinto 2 -en el Espolón 2-, tres en el Sector Central -recintos 35, 36 y 54-y una trinchera -Recinto $55-$. Sobre la base de la cerámica recuperada en el sitio y de las dataciones radio-

\footnotetext{
1 El período Tardío se desarrolló en el noroeste argentino entre los siglos XI y XV y ha sido caracterizado por un fuerte crecimiento demográfico, concentración de poblaciones, territorios controlados y defendidos por sitios en altura y tecnología agrícola con desarrollo de obras hidráulicas.
} 
carbónicas realizadas, que abarcan rangos calibrados desde el siglo XIII al XVI ${ }^{2}$, el Cerro Colorado fue adscrito a los momentos tardíos de ocupación del valle, incluyendo tiempos pre-inkaicos y probablemente inkaicos (Balesta et al. 2011; Wynveldt y López Mateo 2010).

La topografía del Cerro Colorado es irregular y el acceso es difícil por todos los sectores, sobre todo sus laderas este y sur; en los flancos oeste y norte, algo más accesibles, se construyeron murallas defensivas, parapetos y posibles puestos de observación (Wynveldt y López Mateo 2010). El sitio incluye más de 100 recintos y decenas de otros tipos de estructuras de piedra tales como muros y murallas, así como cistas ${ }^{3}$ funerarias actualmente saqueadas. Los recintos se agrupan en dieciocho conjuntos de estructuras contiguas entre sí, que configuran cinco sectores separados a los que se agregan recintos aislados, y fueron construidos con rocas presentes en las inmediaciones (Flores 2013). La circulación entre los diferentes sectores del sitio, ubicados a distintas cotas y en espacios con distintos grados de accesibilidad, implicó la construcción de sendas que debieron estructurar el tránsito dentro del poblado.

No se han registrado espacios centrales grandes. No obstante, algunos recintos se comunican con espacios planos y amplios, probablemente usados para diversos tipos de actividades, similares a las que actualmente desarrolla la población, como la manufactura de textiles, cocina y otras tareas domésticas.

\section{La presencia de maíz en sitios arqueológicos del Noroeste argentino}

Las investigaciones arqueológicas realizadas en varias localidades del noroeste argentino dan cuenta tanto de la antigüedad como del carácter intensivo del cultivo de diversas razas de maíz (Cámara y Rossi 1968; González y Pérez 1972; Cámara 1973, 1989; Cámara y Miante 1989; Pochettino y Scattolin 1991; Fernández Distel et al. 1995; Oliszewski 2004, 2005, 2008, 2009). La presencia de estos cultivos se ha manifestado tanto en valles mesotermales bajos e intermedios -por debajo de los 3.000 m s.n.m.- como en zonas de puna -por encima de los 3.000 m s.n.m.- (Oliszewski 2009), lo que atestigua una diversidad de prácticas en el manejo del recurso.

En cuanto a la identificación de los maíces en sitios arqueológicos, en 1973 Cámara realizó estudios sobre restos que permitieron relacionar el material proveniente de Santa Rosa de Tastil con la raza actual denominada Chaucha. Asimismo, en 1989 Cámara y Miante determinaron marlos provenientes de un basural del Pukara de Tilcara que podían vincularse con ocho razas de maíz actuales. En 1995, Fernández Distel y colaboradores identificaron materiales provenientes de Huachichocana como pertenecientes a las actuales Chaucha, Amarillo Chico y Azul. Otras ocho razas de maíz han sido determinadas en el sitio Pampa Grande por Miante y Cámara (1996)

\footnotetext{
2 Si bien el período Tardío abarca desde el 1000 al 1470 d.C., el análisis de las dataciones radiocarbónicas realizadas por nuestro equipo lleva a ubicar la ocupación de los sitios tardíos principalmente entre los siglos XV y XVI. Por otra parte, las características de la mayoría de los contextos datados permiten sostener que corresponderían a los últimos eventos previos al abandono de esos sitios.

3 Cista es el nombre regional que se aplica a las estructuras funerarias circulares bajo tierra, con paredes y cubierta construidas con piedras, dentro de las cuales se ubicaban los cuerpos y ofrendas acompañantes.
} 
a partir de restos de marlos arqueológicos. Rodríguez y Aschero (2007), en los sitios Punta Peña 4 y Punta Peña 9, identificaron cinco variedades. Para el sitio Cueva de los Corrales 1, Oliszewski (2008) describe la identificación precisa, a partir de los marlos, de dos especímenes: Pisingallo y Culli y con cierta reserva la identificación de otros tres especímenes; a partir de los granos, fueron identificados: Pisingallo, Harinoso y Morocho Amarillo. En 2009 Oliszewski y Olivera publicaron la determinación de ocho subespecies de maíz en los sitios arqueológicos Cueva Cacao 1A y Bajo del Coypar II.

En lo concerniente al valle de Hualfín se pueden citar los hallazgos de A. R. González en el denominado Recinto 45 de Loma de los Antiguos, consistentes en un pozo de almacenaje de $40 \mathrm{~cm}$ de diámetro por $70 \mathrm{~cm}$ de profundidad, tres manos de moler y restos quemados de algarrobo, mazorcas de maíz y maní pelado. Los restos fueron identificados como un trozo de vaina de algarrobo (Prosopis alba. Grise); semillas sueltas y conglomerados de semillas de maní (Arachis hypogaea) quemados y granos de maíz amiláceo y marlos identificados como Zea mays amylacea, Capia (González y Pérez 1968).

Sobre el piso del Recinto 6 de Loma de Ichanga, nuestro equipo recuperó restos de marlos carbonizados (aún en proceso de identificación) junto a cerámica ordinaria y decorada y a restos de techo y vigas quemados, que fueron interpretados como un posible evento de abandono planificado (Valencia et al. 2010).

\section{Evidencias indirectas de explotación agrícola en el valle de Hualfín}

Las evidencias indirectas más claras de agricultura en el valle de Hualfín consisten en las grandes extensiones de andenes o terrazas de cultivo observadas a lo largo de todo el faldeo occidental, al pie del Cordón del Durazno, mencionadas por Weiser (1926), González (1954) y Sempé (1999). Si bien en términos generales se asume que estas estructuras se extienden, al menos, desde la quebrada de Carrizal al norte, cerca del actual poblado de Asampay, hasta la zona de Agua Linda al sur (ver Figura 1), no existe información precisa acerca de la superficie real que abarcan. Se han hallado obras de este tipo en la misma quebrada de Asampay y en las de Carrizal, Cachiyuyo, el Chivo, Chistín y Grande que han sido registradas por el equipo del LAC y contrastadas con imágenes satelitales; algunas de las principales se describen a continuación.

En la quebrada de Asampay, sobre la margen sur del río homónimo, hay restos de un estanque ubicado en la terraza del río y de dos acequias, una de las cuales es utilizada en la actualidad, luego de haber sido acondicionada por pobladores locales. El estanque tiene más de $10 \mathrm{~m}$ de diámetro por $2 \mathrm{~m}$ de profundidad, y está construido con paredes de piedra, cantos rodados superpuestos y, en ocasiones, algún gran bloque de piedra in situ.

Por debajo del nivel de las tomas de agua de la quebrada se observan zonas aterrazadas con paredes frontales de contención. Sobre la superficie del terreno se perciben líneas de pirca extendidas entre barrancas por más de $3 \mathrm{~km}$ de largo, que se van adaptando a la topografía. También se construyeron paredones laterales bordeando los cauces a fin de disminuir el proceso erosivo sobre las barrancas. 


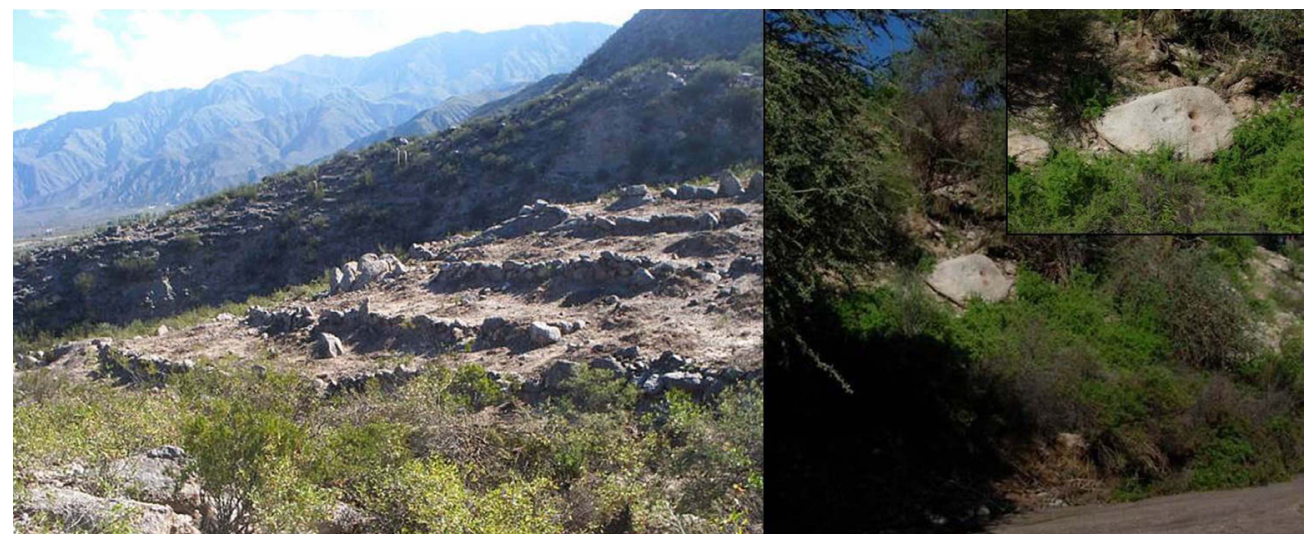

Figura 2: Terrazas del sitio Campo de Carrizal (Carrizal de Asampay) y mortero múltiple identificado en barranca a la vera del río Ichanga.

En la quebrada de Carrizal existen asimismo construcciones aterrazadas con pircas de contención y se observan tres acequias, a distintos niveles, con su respectiva toma de agua. Las acequias presentan rebordes de alrededor de $1 \mathrm{~m}$ de ancho y son usadas en la actualidad como sendas. Sobre la orilla sur hay un estanque de aproximadamente $10 \mathrm{~m}$ de diámetro, que se percibe como una depresión circular del terreno. La salida de agua del estanque conforma una depresión estrecha y alargada, que se ubica en dirección noreste y va bajando con una suave inclinación hacia la zona donde se observan los primeros niveles aterrazados. El estanque podría regular la fuerza del agua que bajaba por la acequia hacia la zona de cultivo, y también dosificar la cantidad de agua para asegurar una provisión constante para el riego, así como para minimizar el lavado del suelo.

En la bajada de las lomas de Carrizal, hacia el este, se han registrado, como mínimo, nueve niveles aterrazados con grandes pircas de contención que siguen las sinuosidades del terreno y varían entre $60 \mathrm{~cm}$ y $1 \mathrm{~m}$ de altura en su parte frontal. Para su construcción se usaron los mismos materiales y técnicas que en Asampay. Los niveles de terraza fueron rellenados, entre paredes, en la modalidad de terraplenes que presentan entre 10 y 20 m de ancho. Los más anchos se encuentran en lugares de pendiente fuerte, lo cual permitiría aprovechar una mayor superficie para el cultivo (Figura 2).

Actualmente en la zona se observan niveles de terrazas similares a las arqueológicas que utilizan el mismo sistema de provisión de agua; tal es el caso de la quebrada Agüita, donde se cultivan vides y frutales.

A $1 \mathrm{~km}$ al noreste de Asampay se hallan las quebradas de Cachiyuyo y el Chivo y aproximadamente a $1 \mathrm{~km}$ al sur de Asampay se encuentra la quebrada de Chistín. Sobre las laderas y campos adyacentes a las mismas se observaron antiguas acequias y niveles aterrazados con paredes de contención de pirca. Asimismo, se registran niveles de terrazas de más de $10 \mathrm{~m}$ de ancho al norte de la quebrada Grande, ubicada unos $4 \mathrm{~km}$ al sur de Asampay.

En la zona de Agua Linda, río Las Mansas y Huasayacu se ven igualmente importantes obras agrícolas, en un área de unos $5 \mathrm{~km}^{2}$. Sobre la margen sur desde la salida 
de la quebrada de Agua Linda se han construido varias líneas de terrazas de cultivo, mientras que en el piedemonte que desciende sobre la margen norte del río Las Mansas, también se observan pircas de contención posiblemente relacionadas con tareas agrícolas, aunque dispuestas de manera dispersa. Estas obras debieron estar vinculadas con la ocupación del Pukara de Huasayacu, emplazado sobre la loma ubicada sobre la banda norte del río Las Mansas.

Otra clase de evidencias de procesamiento de vegetales está representada por distintos tipos de artefactos líticos de molienda, registrados en sitios o próximos a ellos. En la localidad de Carrizal de Asampay, en el sitio Campo de Carrizal, se han documentado dos morteros de granito de gran tamaño con varias oquedades en cada uno de ellos. El de mayor tamaño es una pieza inmóvil, que se encuentra a su vez rodeada por una pared perimetral. Las partes activas de molienda están compuestas por manos de molino y mortero en roca sedimentaria. Entre Campo de Carrizal y Asampay se ha registrado un mortero fijo de granito pircado, de forma poliédrica irregular con varias oquedades.

En el sitio Loma de los Antiguos, de la localidad de Asampay, se cuenta con evidencia sobre 17 artefactos líticos de molienda, distribuidos en diferentes sectores del mismo. En la senda de acceso se halla un mortero fijo sobre un gran bloque de granito que aflora aproximadamente a $30 \mathrm{~cm}$ de la superficie y presenta una forma poligonal irregular, sobre el cual se encuentran abiertas tres oquedades. Además, existe un molino móvil recuperado en un espacio vinculado con otros recintos que ha sido interpretado por Wynveldt (2009) como un «patio» y dentro de los recintos se documentaron partes activas y pasivas de molienda.

Como ya mencionamos, en el Recinto 45 de este sitio se hallaron artefactos líticos de molienda (González y Pérez 1972). Al pie del sitio se han registrado diversos morteros de varias oquedades, realizados sobre bloques planos de granito que alcanzan diámetros de entre 2 y $3 \mathrm{~m}$. Otros morteros presentan paredes, modalidad habitual en la zona de Asampay destinada a proteger de los habituales vientos de la zona.

También en la localidad de Asampay y la quebrada de Cachiyuyo se observaron numerosos morteros comunales, de aproximadamente $2 \mathrm{~m}$ de diámetro, realizados sobre rocas planas aflorantes.

En el sitio Loma de Ichanga, en la localidad de La Ciénaga, se registraron tres morteros de granito. En el trayecto hacia este sitio, sobre las pendientes del cauce del río Ichanga, hay varios morteros fijos de granito de tamaños variables; sobre la barranca sur se ha registrado un mortero fijo configurado sobre un gran bloque, que presenta varias oquedades y se halla en posición inestable, con peligro de desmoronarse por efecto de la erosión (Flores 2013; ver Figura 2).

En la localidad de Agua Linda se observaron morteros con varias oquedades sobre afloramientos rocosos de granito. También en las proximidades del Pukará de Huasayacu se observaron morteros fijos sobre bloques de gran tamaño.

En el Cerro Colorado se encontraron siete artefactos; seis corresponden a morteros y el séptimo está representado por una mano de mortero, recuperada en la superficie del Recinto 46. Todas las partes pasivas registradas hasta el momento corresponden a morteros móviles con una sola oquedad, la mayor parte de ellos confeccionados en granito y hallados en sectores externos a los recintos. En la zona baja adyacente al 
Cerro Colorado y sobre su ladera se registraron varios morteros fijos sobre bloques de granito, con oquedades de distintos tamaños. Sobre la margen izquierda del río también se registraron fragmentos de morteros y manos, algunos de ellos manufacturados en roca volcánica.

Según se ha descrito, las obras hidráulicas y las partes activas y pasivas de molienda configuran una cantidad importante de evidencias indirectas de explotación agrícola, dispersas en una amplia zona del valle.

\section{Las excavaciones en el Recinto 35}

Entre 2008 y 2014 se llevaron a cabo excavaciones en los recintos 35 y 36 (Figura 3 derecha). Estos dos recintos forman parte del denominado Conjunto VIII, en el Sector Central del Cerro Colorado (Figura 3 izquierda). Las medidas del Recinto 35 son de 11 x $9 \mathrm{~m}$; a la superficie delimitada se deben restar unos $22 \mathrm{~m}^{2}$, que corresponden al Recinto 36. El acceso se localiza en el rincón este y presenta forma de pasillo, lo cual constituye una modalidad habitual en el sitio y en el resto de los sitios documentados para el valle.

A partir de las excavaciones realizadas fue posible delimitar tres estratos; el primero comprende desde el nivel 0 hasta $\operatorname{los} 20-30 \mathrm{~cm}$-las variaciones corresponden a cada cuadrícula y a la pendiente del terreno-. El estrato intermedio abarca entre los 30 y $60 \mathrm{~cm}$; a continuación, en función de la pendiente, que desciende hacia el norte, se halló el piso de la habitación, donde comenzó a observarse un cambio en el sedimento, de fino y friable a duro y pedregoso en la medida en que se llegaba al sustrato rocoso. En líneas generales, los primeros hallazgos de materiales tuvieron lugar dentro del segundo estrato, aproximadamente a partir de $30 \mathrm{~cm}$ y continuaron hasta llegar al piso del recinto. Se recuperó abundante cerámica ordinaria y decorada y carbón. En las cuadrículas $\mathrm{C}$ se hallaron abundantes marlos y en la cuadrícula D2 se exhumó una vasijita entera con asas (Figura 4), apoyada de costado.

Por otra parte, se contabilizaron tres hoyos circulares demarcados con piedras, alineados a lo largo de la pared sudoeste, pero no equidistantes a ella y dos hoyos sobre la pared de la entrada al Recinto 36; en algunos de ellos aún se conservaban restos de los postes de sostén que habían contenido. También se registraron once pozos circulares de aproximadamente $60 \mathrm{~cm}$ de diámetro. Nueve de ellos estaban ubicados sobre el sector sudoeste y otros dos pozos sobre la mitad de la pared noroeste; en todos se presentó un sedimento fino y en algunos también se registraron ceniza y fragmentos cerámicos. Uno de los pozos, localizado en la cuadrícula $\mathrm{H} 3$, por su relación con la cuadrícula contigua $-\mathrm{H} 2-$, donde el piso se halla por encima, podría haber funcionado como un fogón que posteriormente fue rellenado. El resto de los pozos, por sus tamaños y particularidades podrían haber servido de apoyo a las vasijas ordinarias cuyas características se comentan en el siguiente acápite.

En las cuadrículas C3 y D3 se recuperó la mayor cantidad de material óseo, mientras que en la línea de cuadrículas F1 a H1 e I1 a K1 se hallaron abundantes restos de materiales líticos, especialmente concentrados en las tres primeras. Las 


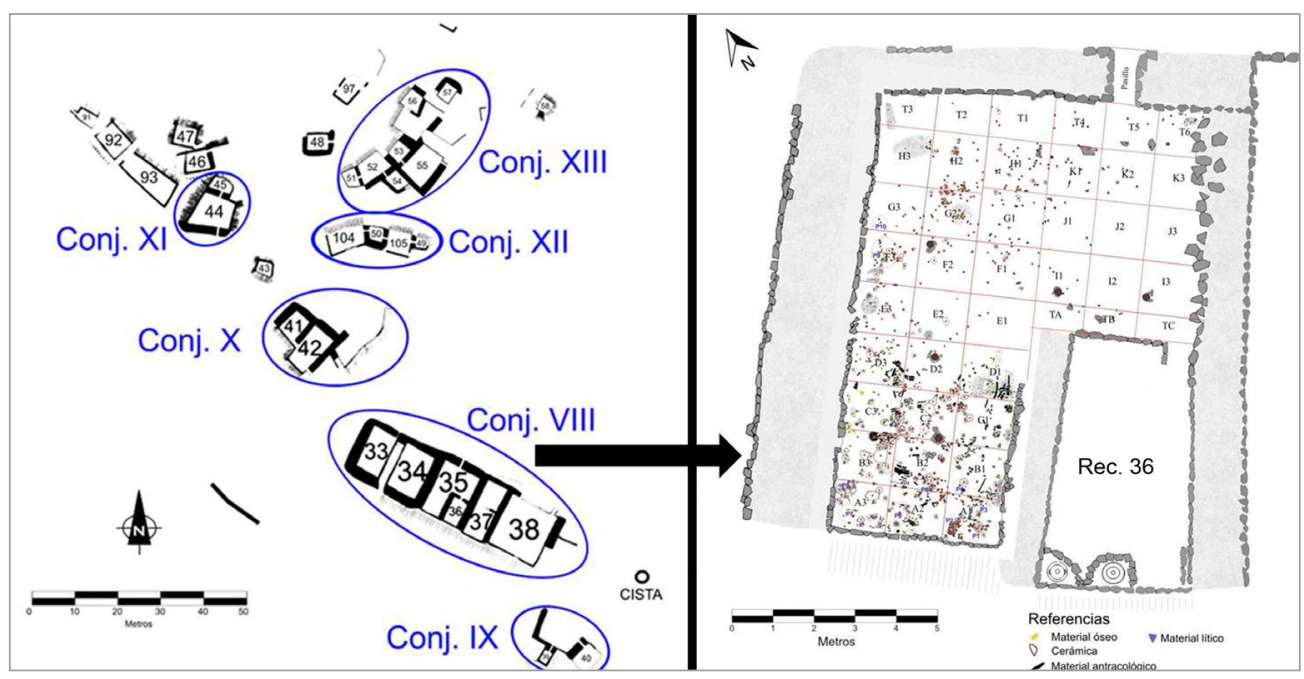

Figura 3: Conjunto VIII del Cerro Colorado y dibujo de la planta de los recintos 35 y 36.

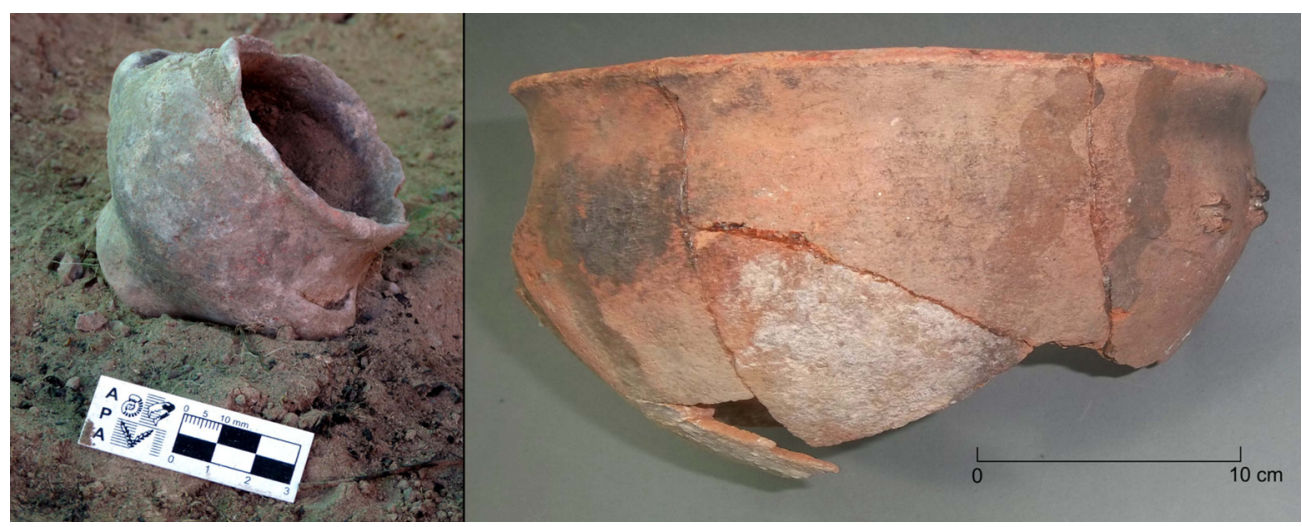

Figura 4: Restos cerámicos hallados en el Recinto 35.

trincheras TA, TB y TC son más pequeñas y se configuraron, para su excavación, teniendo en cuenta que el espacio de la pared que contenía la abertura del Recinto 36 se hallaba muy perturbado por derrumbes, probablemente por acción de raíces. Las cuadrículas alineadas con la entrada del Recinto 35 se hallaban casi limpias de materiales; posiblemente este sector se usara como espacio de circulación permanente.

Las evidencias reseñadas apuntan a interpretar este espacio como un lugar amplio, semicubierto, en el cual se realizarían actividades cotidianas que requirieran tanto de la existencia de lugares descubiertos como resguardados. 


\section{Los hallazgos en el Recinto 35}

\subsection{Cerámica}

En el Recinto 35 se halló cerámica ordinaria y fina; dentro de esta última categoría todos los restos se adjudican al tipo Belén Negro sobre Rojo ${ }^{4}$. Las morfologías correspondieron a cuencos y tinajas, las formas más habituales en estos sitios. Teniendo en cuenta los resultados del remontaje se han contabilizado como mínimo cuatro cuencos, tres tinajas, cinco vasijas ordinarias y una tinaja entera muy pequeña, con estados de conservación variables.

Los cuencos en general presentan un buen nivel de conservación (ver Figura 4) y se han hallado porciones representativas de todas las partes que los componen -base, cuerpo y borde-. Las bases miden entre 6 y $9 \mathrm{~cm}$ de diámetro y las paredes son compactas, con 6 y $7 \mathrm{~mm}$ de espesor a la altura final del borde.

En cuanto a las tinajas, su estado de conservación general es malo y se hallan muy descascaradas. Todas presentan pintura negra sobre rojo, salvo una que además exhibe decoración incisa gruesa. En distintos trozos de tinajas se han contabilizado cinco mamelones in situ y un mamelón desprendido, que se considera que representan como mínimo tres vasijas, ya que según se ha registrado en piezas enteras de colección, cada ejemplar tendría dos mamelones. Cabe señalar el hallazgo de una pequeña tinaja entera, de $5,7 \mathrm{~cm}$ de altura e igual medida en su boca, con un diámetro máximo de $7 \mathrm{~cm}$ y una base de 3,2 $\mathrm{cm}$ (ver Figura 4) y dos asas horizontales en el sector inferior del cuerpo. Sus características de pasta, manufactura y decoración se corresponden con las habituales para la cerámica Belén.

Con respecto a la cerámica ordinaria las alturas medidas comprenden alrededor de 50-60 cm, sólo una es más baja con unos $40 \mathrm{~cm}$ de altura total, y las bocas varían entre 25 y $35 \mathrm{~cm}$. El grosor de las paredes ronda en aproximadamente $1 \mathrm{~cm}$, con excepción de la vasija más baja, que presenta paredes más delgadas con $7 \mathrm{~mm}$ de espesor. Las bases presentan forma cóncavo-convexa y plana; una de ellas tiene un pie de 4 $\mathrm{cm}$ de altura. Varias de las piezas ordinarias exhiben descascarados en sus superficies y en algunos casos el desgranamiento de la pasta, situación que impide su remontaje. Sólo una de ellas muestra hollín en sus paredes.

Se calcularon los volúmenes para las vasijas ordinarias teniendo en cuenta las medidas disponibles y usando referencias de medidas de vasijas semejantes provenientes del Cerro Colorado y de varios sitios del valle (Iucci 2013). Los volúmenes estimados cubren un rango de 40 a 70 litros por pieza en función de sus distintos tamaños.

\subsection{Arqueofauna}

Los estudios sobre la arqueofauna del Recinto 35 se hallan aún en proceso. No obstante, los resultados preliminares indican que se recuperaron 555 especímenes óseos, de

\footnotetext{
4 El tipo Belén Negro sobre Rojo corresponde a vasijas abiertas (cuencos) y cerradas (ollas) sensu Balfet et al. (1992), pintadas con guardas geométricas y/o icónicas en negro sobre una capa de pintura roja, cuyo origen se adscribe al propio valle de Hualfín.
} 
los cuales 15 se identificaron como artiodáctilos, 32 como camélidos y 1 espécimen pudo ser asignado a Vicugna vicugna (Lorenzo 2015). Además, se han identificado restos de aves indeterminadas, mamíferos pequeños como el Thylamys pallidior, y microvertebrados pertenecientes al orden Rodentia.

El 88,15\% de los especímenes asignados a artiodáctilos y camélidos presentan estadios de meteorización bajos que permiten inferir que para la mayoría de los restos se dio un enterramiento relativamente rápido. Respecto a otro tipo de modificaciones de origen natural, sólo un espécimen presenta tanto marcas de raíces como de carnívoro, y otro tiene huellas de dientes de roedor. Debido al bajo porcentaje en el que aparecieron huellas de este tipo, es posible considerar que estos agentes no tuvieron un alto impacto en la muestra.

Con respecto a la representación de partes óseas, en este recinto es posible observar una variedad de elementos anatómicos, con una ausencia notable de los elementos relacionados con el esqueleto axial (específicamente esternón, costillas y vértebras en general), los cuales probablemente estén incluidos dentro de la categoría más amplia de «mamífero grande». Se identificaron marcas de corte en restos asignados a dicha categoría de mamífero grande, mientras que un $12,5 \%$ de los especímenes presenta huellas de termoalteración (quemado, carbonizado y calcinado).

\subsection{Materiales líticos}

En el Recinto 35 se han recuperado 170 artefactos líticos, los cuales aún se encuentran en estudio; la mayoría corresponde a artefactos tallados. En ese conjunto predomina la presencia de rocas sedimentarias locales y disponibles dentro del mismo cerro (macroscópicamente correspondientes a sublitoarenita, feldarenita y litoarenita), seguidas en mucha menor proporción por obsidianas procedentes de más de $100 \mathrm{~km}$ de distancia (macroscópicamente correspondientes a obsidianas de las fuentes Ona y Cueros de Purulla).

La clase tipológica más representada corresponde a desechos de talla, seguidos por artefactos formatizados, núcleos y percutores en menor proporción. Entre los artefactos formatizados se observaron principalmente raederas, raspadores y artefactos de formatización sumaria. Por otra parte, se han encontrado en este recinto tres cantos rodados de feldarenita, de los cuales dos presentan evidencia de haber sido usados posiblemente como manos de morteros. Es interesante señalar la presencia de un mortero en sublitoarenita, realizado a partir de la abertura de una oquedad pequeña en un bloque de morfología cuadrangular.

A partir del análisis preliminar de los hallazgos es posible plantear que el recinto se usaba en la manufactura de artefactos líticos, principalmente con materias primas locales e inmediatas, y en actividades de molienda.

\section{Los maíces}

En Argentina existen al menos 52 razas de maíz, de las cuales 28 se cultivan en el noroeste argentino. Esta amplia variedad pertenece a una misma especie, Zea mays; 
los tipos que los diferencian no corresponden a una clasificación botánica, sino a una utilitaria basada en una multiplicidad de formas, tamaños, colores y texturas y a la adaptación a diferentes ambientes. Tal como expusieran Cámara et al. (2012), los hallazgos de restos de maíz en el registro arqueológico proveen evidencias que indican una estrecha relación entre éstas y las razas andinas peruanas y bolivianas, así como también exponen la existencia de variaciones locales.

Oliszewski $(2008,2012)$ propone una clasificación de los maíces en tipos basándose en las características de los granos y su maduración, de la cual hacemos uso más adelante para describir las razas identificadas en los restos del Recinto 35:

1. Maíces que presentan un endosperma duro, denominados reventadores. Son de maduración temprana y como su ciclo es corto brindan la posibilidad de obtener más de una cosecha anual. No requieren cantidades importantes de agua.

2. Maíces de endosperma semiduro de maduración temprana.

3. Maíces de endosperma blando, que se raya fácilmente con la uña aún cuando el grano no esté maduro y listo para cosechar. Son maíces de maduración tanto intermedia como tardía.

4. Maíces de endosperma azucarado: son dulces y de maduración tardía.

Cada uno de estos tipos requiere de tiempos y condiciones diferentes para su maduración, lo cual brinda la posibilidad de cultivarlos en distintos momentos y espacios.

\subsection{Metodología para la determinación de maíces}

Los aportes a la metodología para la identificación taxonómica de razas de Zea mays en Argentina incluyen las propuestas de Parodi (1959), Abiusso y Cámara (1974) y Cámara et al. (2012), quienes plantearon métodos sistemáticos cuantitativos para ello. Parodi (1959) formuló una metodología basada en el análisis de los caracteres externos de los cariopsis (granos), según la cual identifica ocho variedades. Por su parte, Abiusso y Cámara (1974) y Cámara et al. (2012) proponen la identificación taxonómica basándose en una serie de caracteres tanto externos como internos de la espiga y los caracteres externos del cariopsis. Dichos autores identificaron, en el noroeste argentino, veintiocho razas. En el caso que nos ocupa, dadas las características de las muestras, consistentes en marlos sin cariopsis, hemos adaptado la metodología propuesta por Abiusso y Cámara (1974) y Cámara et al. (2012) para la identificación taxonómica, basándonos en los caracteres externos e internos del marlo, así como en caracteres externos del grano determinados en forma indirecta.

En el Recinto 35 se halló un número mínimo de 53 marlos de maíz, todos termoalterados, de los cuales se seleccionó una muestra de 31 marlos, que comprende aquéllos que se recuperaron enteros. La identificación de las muestras se realizó a ojo desnudo y con lupa binocular a 2X y 4X. Los caracteres morfológicos analizados, teniendo en cuenta las características observables en la muestra, y los resultados del análisis se muestran en el Cuadro 1.

Debido a que la muestra analizada se presenta termoalterada se llevó a cabo una experimentación que consistió en la carbonización de maíz actual con el fin de poder medir, en términos cuantitativos, la alteración sufrida por los materiales durante la 
Cuadro 1: Caracteres morfológicos de la espiga en las muestras analizadas

\begin{tabular}{|c|c|c|c|c|c|c|c|c|c|c|}
\hline & \multicolumn{4}{|c|}{ Caracteres internos del marlo } & \multicolumn{5}{|c|}{ Caracteres externos del marlo } & \multirow{3}{*}{ :气气 } \\
\hline & \multicolumn{3}{|c|}{ Diámetro } & \multirow{2}{*}{. } & \multirow[b]{2}{*}{ 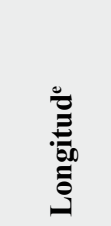 } & \multirow[b]{2}{*}{ 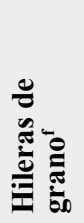 } & \multirow{2}{*}{ 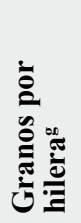 } & \multirow{2}{*}{ 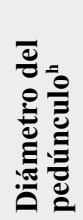 } & \multirow{2}{*}{ 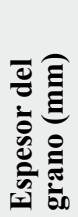 } & \\
\hline & 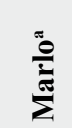 & 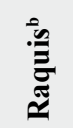 & 莺 & & & & & & & \\
\hline 21 & 2,20 & 1,20 & 0,40 & 1,83 & 8,10 & 15 & 29 & 1,20 & 2,70 & Pisingallo \\
\hline 22 & 2,30 & 1,20 & 0,48 & 1,91 & 9,70 & 14 & 31 & & 3,10 & Pisingallo \\
\hline 6 & 1,70 & 1,20 & 0,59 & 1,54 & 8,60 & 17 & 21 & & 4,09 & Chullpi \\
\hline 14 & 2,00 & 1,20 & 0,61 & 1,66 & 7,80 & 14 & 18 & & 4,30 & Chullpi \\
\hline 20 & 1,70 & 0,90 & 0,60 & 1,60 & 7,00 & 10 & 19 & 1,10 & 4,20 & Garrapata \\
\hline 4 & 1,85 & 1,20 & 0,45 & 1,54 & 12,00 & 12 & 34 & & 3,50 & Amarillo Grande \\
\hline 10 & 1,60 & 0,90 & 0,30 & 1,77 & 9,50 & 10 & 30 & & 3,10 & Amarillo Chico \\
\hline 23 & 1,57 & 0,83 & 0,31 & 1,89 & 9,50 & 14 & 33 & & 2,80 & Amarillo Chico \\
\hline 24 & 1,48 & 0,85 & 0,35 & 1,74 & 8,00 & 14 & 32 & & 2,80 & Amarillo Chico \\
\hline 2 & 1,60 & 1,00 & 0,40 & 1,60 & 8,63 & 15 & 28 & 7,00 & 3,00 & Culli \\
\hline 19 & 1,48 & 0,90 & 0,38 & 1,64 & $(+) 7,00$ & 11 & 25 & & 2,80 & Cf. Culli \\
\hline 26 & 1,50 & 0,95 & 0,40 & 1,57 & $(+) 7,80$ & 10 & 27 & & 2,88 & Cf. Culli \\
\hline 29 & 1,60 & 0,70 & 0,40 & 2,28 & $(+) 5,60$ & 10 & 22 & & 2,50 & Cf. Azul \\
\hline 9 & 1,50 & 0,75 & 0,30 & 2,00 & 7,30 & 11 & 29 & & 2,50 & Azul \\
\hline 27 & 1,50 & 0,80 & 0,37 & 1,87 & 6,50 & 11 & 28 & & 2,30 & Azul \\
\hline 30 & 2,20 & 1,60 & 0,70 & 1,37 & 9,00 & 15 & 26 & & 3,00 & Capia \\
\hline 16 & 2,00 & 1,00 & 0,70 & 1,53 & 9,50 & 15 & 28 & & 2,80 & Capia \\
\hline 17 & 2,40 & 1,65 & 0,80 & 1,45 & 9,50 & 17 & 26 & & 3,00 & Capia \\
\hline 18 & 1,67 & 1,50 & 0,58 & 1,11 & $(+) 6$ & 14 & 22 & & 2,70 & Cf. Capia \\
\hline 28 & 1,40 & 0,95 & 0,35 & 1,47 & 9,00 & 17 & 35 & & 2,50 & Indet. \\
\hline
\end{tabular}

${ }^{a}$ Medida entre el extremo distal de la gluma inferior de un grano y el extremo distal del grano opuesto.

${ }^{\mathrm{b}}$ Diámetro medido entre la base de la gluma inferior de un grano y la base de la gluma inferior del grano opuesto

${ }^{\mathrm{c}}$ Diámetro de la médula

d Índice obtenido diviendo el diámetro del marlo entre el diámetro del raquis

${ }^{e}$ Longitud en centímetros desde la base hasta el ápice de la muestra.

${ }^{\mathrm{f}}$ Número de hileras de grano en la parte media de la espiga.

${ }^{g}$ Número de granos en una hilera; este número permite establecer el espesor relativo de los granos en caso de falta de los mismos.

${ }^{\text {h }}$ Diámetro del pedúnculo en centímetros en el eje de la espiga.

carbonización. Las muestras de maíz actual se carbonizaron en un horno de gas con control de temperatura. Se utilizaron marlos (espiga sin granos) que fueron protegidos con papel aluminio a fin de evitar que entraran en contacto con el oxígeno durante la carbonización, para evitar la posibilidad de que el material se convirtiese en cenizas. El maíz termoalterado nos proporcionó información útil acerca de cómo afecta el fuego a estos materiales. A partir de la experimentación hemos podido verificar que ciertos caracteres cuantitativos varían porcentualmente en el marlo sin carbonizar con respecto al marlo carbonizado. Esta variación porcentual corresponde al valor final (marlo carbonizado) menos el valor inicial (no carbonizado) multiplicado por 100 y dividido por el valor inicial. Con base en estas variaciones, los caracteres cuantitativos a tener en cuenta, en lo que refiere a la identificación racial de maíces 
Cuadro 2: Variación de los caracteres cuantitativos en el marlo sin carbonizar con respecto al marlo carbonizado

\begin{tabular}{lccrrr}
\hline \multicolumn{1}{c}{ Razas } & \multicolumn{2}{c}{ Diámetro } & \multicolumn{2}{c}{ Longitud } & Índice marlo/raquis \\
\cline { 1 - 4 } (carb.)
\end{tabular}

arqueológicos carbonizados son los siguientes (Cuadro 2): longitud del marlo, que se reduce un $18 \%$ en el marlo carbonizado; diámetro del marlo, que se reduce un $20 \%$ en el marlo en las mismas circunstancias; es de destacar que, a pesar de la reducción en diámetro, el índice marlo/raquis se mantiene estable.

\subsection{Resultados de la determinación de los maíces}

Dada la variabilidad de los caracteres cuantitativos diagnósticos presentes en las muestras analizadas, sumado al empleo de la variación porcentual de los caracteres morfológicos cuantitativos, hemos podido distinguir lo que denominamos como diez tipos, de los cuales ocho han podido identificarse y relacionarse con razas actuales (Figura 5).

Grupo 1: Pisingallo. La espiga posee de 14 a 20 hileras de granos medianos no dentados y córneos. Su forma es cilíndrica de $11,3 \mathrm{~cm}$ de longitud y el diámetro del marlo es de aproximadamente unos $24,8 \mathrm{~mm}$. Se cultiva en las provincias de Jujuy, Salta, Santiago del Estero, La Rioja y Catamarca. Cámara et al. (2012) han propuesto que podría relacionarse con las formas raciales de Bolivia llamadas Perla Blanco, Grande y Perla, integrantes del Complejo Racial Perla. Otros autores como Rodríguez et al. (1968, en Cámara et al. 2012) consideran que es posible que en el origen de este complejo haya tenido participación el maíz Pisanckalla de Bolivia. El Complejo Racial Pisanckalla corresponde a maíces reventadores de granos pequeños y muy duros distribuidos en los valles templados interandinos en alturas entre 2.000 y 3.500 m s.n.m. Se lo considera derivado o relacionado con el grupo Confite puntiagudo de Perú. Pisingallo es una raza de maduración temprana y endosperma duro, que no tiene grandes requerimientos respecto al agua (Oliszewski 2012). Se utiliza actualmente en la elaboración de platos guisados tales como «frangollo», aloja, 

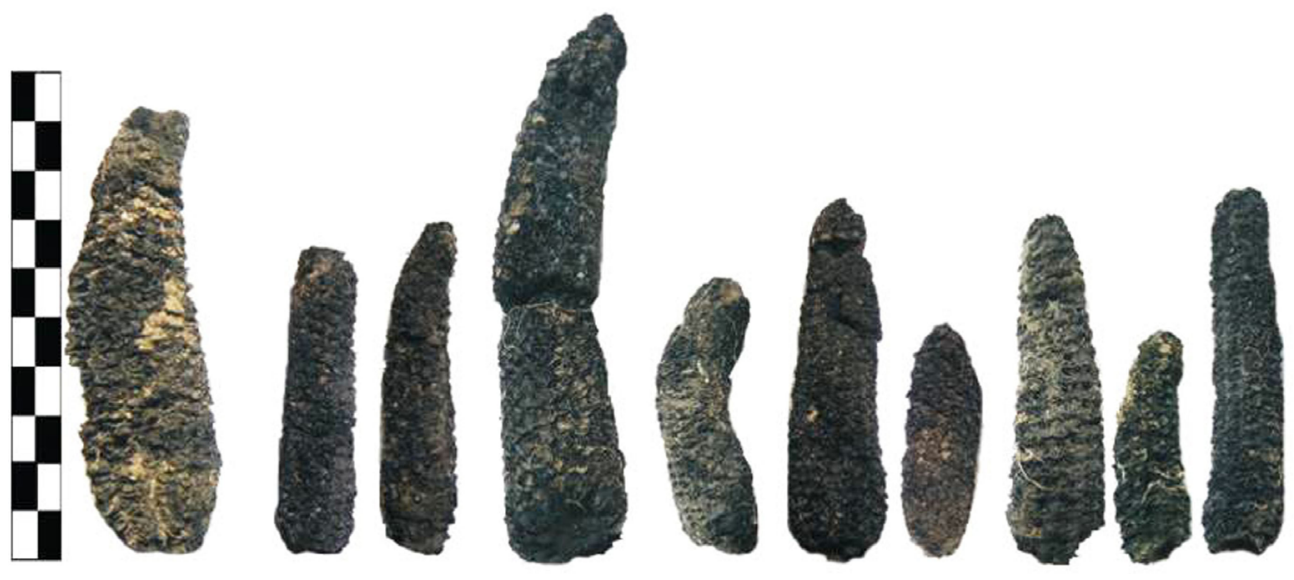

Figura 5: Distintas variedades de maíces recuperadas en el Recinto 35. De izquierda a derecha: Pisingallo, Chullpi, Amarillo Chico, Amarillo Grande, Capia, Culli, Garrapata y Azul; los dos últimos ejemplares no fueron identificados.

mazamorra y locro, para hacer pochoclo y un tipo particular de harina a la que se la denomina «harina cocida».

Grupo 2: Chullpi. Presenta 18 a 24 hileras de granos largos, angostos y delgados, de forma aovada o cónica de 10,4 cm de longitud, con un diámetro de marlo de alrededor de $26 \mathrm{~mm}$. Se cultiva en Jujuy, Salta, Tucumán y Catamarca. Se asemeja a las razas Chullpi de Perú y Chuspillo de Bolivia. Su centro de dispersión está localizado en los departamentos del Cuzco, Apurímac, Huancavelica y Ayacucho, donde se lo encuentra en su forma más pura. En los Andes Peruanos se lo cultiva entre los 2.400 y 3.400 m s.n.m. El Chuspillo de Bolivia, pertenece al Complejo Racial Harinoso del Valle, distribuido entre los 1.500 y 3.000 m s.n.m. (Ávila et al. 1998). Debido a esta semejanza con las razas de Bolivia y Perú, Cámara et al. (2012) postulan que es originaria de aquellas regiones y que su cultivo debe haberse extendido durante épocas del dominio inkaico. Chullpi es de maduración tardía; requiere de abundante agua para su crecimiento (Oliszewski 2012), su endosperma tiene azúcares que le dan sabor dulce; por tal razón sus granos frescos se utilizan para hacer las humitas y el «guajcha locro». Para su conservación durante el invierno se usan los granos maduros, secos, que se suelen tostar y se comen salados. Este maíz es de muy buena calidad para la fabricación de chicha. Según afirma Nicholson (1960), la raza Chullpi del Perú, era una de las preferidas para este fin en la región de Cuzco, cultivada antiguamente y en la actualidad en el valle de Urubamba.

Grupo 3: Garrapata. Presenta 14 a 22 hileras de granos, a veces irregulares, una espiga de forma cilíndrico-cónica de $8,3 \mathrm{~cm}$ de longitud y un diámetro del marlo de $25 \mathrm{~mm}$. Actualmente se cultiva en Jujuy y Salta -aproximadamente a los $3.000 \mathrm{~m}$ s.n.m.- y se ha propuesto que su origen podría ser boliviano y que se correspondería a la raza Checchi descrita por Ramírez (1960, en Cámara et al. 2012) que corresponde al Complejo Racial Harinoso del Valle. Su maduración es tardía y tiene altos requerimientos en cuanto a humedad (Oliszewski 2012). Forma parte de los maíces 
llamados Capia, empleados por su endosperma harinoso, y se utiliza para su consumo directo y para la fabricación de harina tostada, ulpada (bebida a base de harina cocida, agua y azúcar), locro, humita y chilpan.

Grupo 4: Amarillo Grande. Su espiga presenta unas 8 a 10 hileras de granos, es de forma cilíndrica, con una longitud de $14 \mathrm{~cm}$ y unos $20,6 \mathrm{~mm}$ de diámetro. Se cultiva en las provincias de Jujuy y Salta. Esta raza habría surgido por cruzamiento de $\mathrm{Ama}$ rillo chico con Harinoso y se la relaciona con las razas Kellu de Bolivia y Uchuquilla de Perú. La primera pertenece al Complejo Racial Morocho, que se caracteriza por colonizar áreas entre 1.000 y $3.000 \mathrm{~m}$ s.n.m., con bajos niveles de precipitación pluvial y por lo general sin riego (Ávila et al. 1998). La raza Uchuquilla de Perú se cultiva en la zona de Puno, alrededor de los 2.000 a 2.500 m s.n.m. y en una extensión muy limitada del Cuzco. Es una raza de maduración temprana, de endosperma semiduro. Ramos et al. (2013) mencionan su uso en la provincia de Jujuy para la fabricación de chicha. También se lo utiliza para consumo directo y para la fabricación de harina cocida, una bebida denominada anchi, mote y como forraje para los animales.

Grupo 5: Amarillo Chico. Tiene una espiga con 8 hileras de granos que presentan longitud y ancho similares y de espesor delgado, de $11 \mathrm{~cm}$ de longitud y $18 \mathrm{~mm}$ de diámetro. Se cultiva en las provincias de Jujuy, Salta y Catamarca. Cámara et al. (2012) lo vincula, por la similitud en la forma y otros caracteres coincidentes con Kellu de Bolivia y Uchuquilla de Perú, ya que las tres razas presentan caracteres coincidentes. Es una raza de maduración temprana y endosperma semiduro. Ramos et al. (2013) y Cámara et al. (2012) mencionan, para Amarillo Chico, los mismos usos que para Amarillo Grande.

Grupo 6: Culli. Tiene una espiga con 10 a 12 hileras, a veces irregulares, de granos cortos medianamente anchos y gruesos, de $10 \mathrm{~cm}$ de longitud y un marlo de $21 \mathrm{~mm}$ de diámetro. Se cultiva en las provincias de Jujuy y Salta, entre los 1.500 y $3.000 \mathrm{~m}$ s.n.m. Sus caracteres lo relacionan con la raza Kulli Chojnocollo de Bolivia, perteneciente al Complejo Racial Harinoso del Valle. Se considera como uno de los de mejor calidad para la fabricación de chicha, especialmente para la preparación de Chicha Morada. Al igual que Chullpi, ha sido utilizado en la región del Cuzco con este fin y se ha cultivado en el valle de Urubamba hasta la actualidad (Nicholson 1960). Según Abiusso y Cámara (1974) su maduración es medianamente temprana. Esta raza pertenece al grupo de los maíces de endosperma blando harinoso, de maduración intermedia. Es utilizada en la actualidad para la fabricación de chicha, anchi, una bebida denominada api y también tiene uso medicinal.

Grupo 7: Azul. Su espiga tiene 12 hileras de granos, unos $10,3 \mathrm{~cm}$ de longitud y $17,5 \mathrm{~mm}$ de diámetro; de marlo y raquis delgados, raquilla larga y grano muy poco cubierto de glumas. Se cultiva en la provincia de Jujuy; Cámara et al. (2012) señalan que su origen provendría de la dispersión de material boliviano. Es un maíz semiduro de maduración intermedia (Oliszewski 2012) que se cultiva entre los 2.500 a $3.500 \mathrm{~m}$ s.n.m. Se emplea principalmente para producir alimentos sobre la base de la harina; en Bolivia y Perú también se lo usa para hacer Chicha Morada.

Grupo 8: Capia. Presenta una espiga con 14 a 18 hileras de granos, de forma cilíndrico-cónica y $12,5 \mathrm{~cm}$ de longitud y un marlo de $29 \mathrm{~mm}$ de diámetro. Es un maíz amiláceo que se cultiva en las provincias de Salta, Jujuy, Catamarca y La 
Rioja. Cámara et al. (2012) consideran que esta raza se habría originado por el cruzamiento de Harinoso con Chullpi y que este cruzamiento se habría dado en Jujuy. Es un maíz de endosperma blando, de maduración tardía. Algunos autores ubican su cultivo a partir de los 2.000 m s.n.m. (Oliszewski 2012; Ramos et al. 2013). Se utiliza para su consumo directo y para la elaboración de tortillas, bollos y humitas (Ramos et al. 2013).

\section{Discusión}

La identificación de ocho variedades utilizadas en el Cerro Colorado revela, por un lado, el cultivo de una importante diversidad de maíces, que incluyen tanto razas de maduración temprana como tardía. Como indicáramos, algunas de las razas de maíz identificadas poseen influencias de otras andinas, ya sea de Perú o Bolivia, mientras que algunas habrían surgido en el noroeste argentino por cruzamiento. Todas las variedades reconocidas se cultivan actualmente en el noroeste argentino, aunque con desigual intensidad.

Las variedades de maíces registradas se pueden cultivar a distintas cotas. La altura a que se encuentra el Cerro Colorado, así como las evidencias de cultivo registradas en áreas aledañas, permiten obtener todas las variedades, por lo cual existe la posibilidad de que fueran cultivadas en la propia zona. Este planteamiento no descarta, sin embargo, el ingreso de maíces por vía de intercambio con otros grupos, lo cual contribuiría a ampliar la oferta del recurso.

Si bien las evidencias arqueológicas de áreas agrícolas no se ubican en la propia área del Cerro Colorado, actualmente existen muchas fincas en la zona baja adyacente al mismo que aprovechan la cercanía del río para la agricultura; es probable que estas zonas hayan sido utilizadas también en el pasado con la misma finalidad. Por otra parte, como ya hemos comentado, a pocos kilómetros del Cerro Colorado se halla una serie de obras hidráulicas asociadas a zonas agrícolas, que indican -por la amplia superficie cubierta- que de allí se podría haber obtenido una parte sustancial de los recursos consumidos tanto en el sitio como en toda la zona.

La variabilidad y abundancia del recurso maíz, sumado a las áreas de cultivo que se observan tanto en los alrededores del sitio como en las inmediaciones, nos llevan a plantear la hipótesis de que en el área habría una multiplicidad de formas de manejo y de prácticas tanto para la siembra y la cosecha como para la protección de los cultivos, que permitirían la obtención de morfotipos determinados, según estrategias planificadas, acordes con pautas de consumo que obedecerían a gustos particulares y a necesidades vinculadas a la cantidad de población existente.

La detección del cultivo y el consumo de distintos tipos de maíz (duros, semiduros, harinosos y dulces) nos da la pauta de que estos grupos implementaban actividades y tecnologías variadas para la explotación de condiciones ambientales diferentes. Por otra parte, el registro de razas de maduración temprana (Pisingallo, Amarillo chico y Amarillo grande), intermedia (Culli y Azul) y tardía (Garrapata, Chullpi y Capia), implican que habrían sido cosechadas en diferentes momentos, lo que proporcionaría la posibilidad de obtener recursos frescos durante todo el año. 
El contexto en que se hallaron los marlos nos remite a un espacio semicubierto de dimensiones considerables, que albergaba no sólo el maíz sino también restos óseos de animales -con predominio de camélidos y mamíferos grandes-, manos de moler y un mortero; a ello se suma la presencia de al menos un fogón, varios pozos y recipientes cerámicos. Por todo ello se lo ha interpretado como un lugar vinculado al procesamiento de alimentos, entre los cuales se incluiría la producción de chicha al nivel de una o varias unidades domésticas (Balesta et al. 2014).

Como hemos visto, los distintos maíces también se diferencian por los usos a los que se someten según sus características, que posibilitan la implementación de preparaciones diferentes: dulces o saladas, guisos, sopas, papillas, bebidas. Estos usos diversos implican la realización de actividades específicas, como molido, calentado, hervido y tostado.

Las características espaciales del recinto y los materiales identificados en él (marlos, tinajas, áreas cubiertas y descubiertas, pozos, fogones y combustible) se corresponden con correlatos de fabricación de chicha detectados en tiempos prehispánicos, tanto en el Área Andina Central como en el noroeste argentino (Moore 1989; Cremonte et al. 2009). A esto se suma que dos de los tipos de maíz registrados -Culli y Chullpi, que representan un 25\% de la muestra- han sido citados (Nicholson 1960) dentro de los más utilizados y valorados para hacer chicha.

Las evidencias materiales recuperadas nos hablan de prácticas tanto individuales como colectivas en las que podrían participar y relacionarse varias personas a la vez, mientras que su localización indica el uso de diferentes espacios, internos y externos a los recintos, para el desarrollo de estas tareas (Flores 2013). Por otra parte, estos restos y prácticas también son indicadores de género; las actividades de elaboración de alimentos, entre ellas la molienda, han sido vinculadas con las mujeres (Babot 2007), del mismo modo que el procesamiento de maíces para la fabricación de chicha (Cremonte et al. 2009).

Los datos aquí presentados dan cuenta, por primera vez en el área, de la presencia de distintas variedades de maíz que nos indican el conocimiento de recursos y tecnologías puestos en marcha por estos grupos para producir alimentos. También se manifiestan actividades culinarias que reúnen a varias personas en espacios definidos, donde socializan y se involucran en diversas tareas. A través de estas prácticas, sus participantes no sólo podrían incorporar ingredientes y herramientas para cocinar sino también temporalidades y ritmos cotidianos que contribuirían a forjar y manipular identidades y modos de pensar, de sentir, de percibir e imaginar sus lugares en el mundo.

Agradecimientos: A la Universidad Nacional de La Plata, por brindar financiamiento e infraestructura para la realización de los trabajos. A la Agencia de Promoción Científica y Tecnológica; a través de la financiación de los PICT 38174 y 0551 se llevaron a cabo tareas de campo y se realizaron dataciones radiocarbónicas.

\section{Referencias bibliográficas}

Abiusso, Noemí y Julián CÁmara HernándeZ

1974 «Los maíces autóctonos de la Quebrada de Humahuaca (Jujuy), sus niveles nitrogenados y su composición en aminoácidos». Revista de la Facultad de Agronomía 50: $1-25$. 
Ávila, Gonzalo, Lorena GuZmán y Marcia CÉsPedes

1998 Catálogo de recursos genéticos de maíces bolivianos conservados en el Banco de Germoplasma del centro de investigaciones del Centro Fitoecogenéticas de Pairumani. Bolivia: FONAMA.

BАвот, Pilar

2007 «Organización social de las prácticas de molienda: casos actuales y prehispánicos del Noroeste Argentino», en Procesos sociales prehispánicos en el sur andino, Axel Nielsen, ed., pp. 259-290. Córdoba: Editorial Brujas.

Balesta, Bárbara, Celeste Valencia y Federico Wynveldt

2014 «Procesamiento de maíz y producción de chicha en el Tardío del valle de Hualfín ¿Un contexto doméstico de producción de chicha?». Revista Arqueología 20 Dossier 2014: 13-36.

BALESTA, Bárbara, Nora ZAGORODNY y Federico Wynveldt

2011 «La configuración del paisaje Belén (Valle de Hualfín, Catamarca, Argentina)». Relaciones de la Sociedad Argentina de Antropología 36: 149-175.

Balfet, Helene, Marie France Fauvet-Berthelot y Susana Monzón

1992 Normas para la descripción de vasijas cerámicas. México: Centre d'Etudes Mexicaines et Centramericaines.

Bruch, Carlos

1911 «Exploraciones arqueológicas en las provincias de Tucumán y Catamarca». Revista del Museo de La Plata XIX, primera parte (2 $2^{\mathrm{a}}$ serie, VI).

CÁMARA HERnÁNDEZ, Julián

1973 Restos arqueológicos de maíz de Santa Rosa de Tastil (Salta)», en Tastil, una ciudad preincaica argentina, Eduardo Cigliano, ed., pp. 559-564. Buenos Aires: Camargón.

1989 «Restos arqueológicos de maíz (Zea mays L.) de León Huasi, provincia de Jujuy, Argentina». Comunicaciones Científicas 1: 18-26. San Salvador de Jujuy.

CÁmARA Hernández, Julián y Juan Carlos Rossi

1968 «Maíz arqueológico de Cafayate, Salta». Boletín de la Sociedad Argentina de Botánica XII: 234-242.

Cámara Hernández, Julián y Ana María Miante Alzogaray

1989 «Maíz (Zea mays ssp. mays) arqueológico del Pucará de Tilcara, Jujuy, Argentina». Revista de la Facultad de Agronomía 10 (3): 99-108.

Cámara Hernández, Julián, Ana María Miante Alzogaray, Ricardo Bellón y Ana Julia GALMARINI

2012 Razas de maíz nativas de la Argentina. Buenos Aires: Facultad de Agronomía, Universidad Nacional de Buenos Aires.

Caminos, Roberto

1979 «Sierras Pampeanas Noroccidentales. Salta, Tucumán, Catamarca, La Rioja y San Juan». $2^{\circ}$ Simposio de Geología Regional Argentina. Academia Nacional de Ciencias I, pp. 225-291.

Cremonte, Beatriz, Clarisa Otero y Soledad Gheggi

2009 «Reflexiones sobre el consumo de chicha en épocas prehispánicas a partir de un registro actual en Perchel (Dto. Tilcara, Jujuy)». Relaciones de la Sociedad Argentina de Antropología 34: 75-102. 
Fernández Distel, Alicia, Julián CÁmara Hernández y Ana María Miante Alzogaray 1995 «Estudio del maíz arqueológico de Huachichocana 2, provincia de Jujuy, Noroeste de Argentina». Relaciones de la Sociedad Argentina de Antropología 20: 101-127.

FLORES, Marina

2013 Aprovisionamiento y manejo de materias primas líticas durante el Periodo de Desarrollos Regionales/Inka en el Valle de Hualfin (Catamarca. Argentina). Tesis doctoral inédita. Facultad de Ciencias Naturales. La Plata.

Garcilaso De La Vega, El Inca

1800 Historia general del Perú, o, comentarios reales de los Incas. Madrid: Imprenta del Villalpando.

GonzÁLEZ, Alberto Rex

1954 «Contextos Culturales y Cronología Relativa en el Área Central del NOA». Anales de Arqueología y Etnología 11: 7-32.

GonzÁLEZ, Alberto Rex y José Antonio Pérez

1968 «Una nota sobre etnobotánica del N. O. Argentino». Actas y Memorias. XXXVII Congreso Internacional de Americanistas, vol. 2, pp. 209-234. Buenos Aires.

1972 Argentina indígena: vísperas de la conquista. Buenos Aires: Paidós.

Guaman Poma De Ayala, Felipe

1987 Nueva corónica y buen gobierno. [1615], Rolena Adorno, John V. Murra y Jorge L. Urioste, eds. Madrid: Historia 16.

Hastorf, Christine y Sissel Johanessen

1993 «Prehispanic Political Change and the Role of Maize in the Central Andes of Peru». American Anthropologist 95 (1): 115-138.

HAYASHIDA, Frances

2008 «Chicha Histories. Pre-Hispanic Brewing in the Andes and the Use of Ethnographic and Historical Analogues», en Drink, Power, and Society in the Andes, Justin Jennings y Brenda Bowser, eds., pp. 232-256. Gainsville: University Press of Florida.

IUCCI, María Emilia

2013 Producción, uso y circulación de cerámica durante el Período de Desarrollos Regionales en el Valle de Hualfín. Tesis doctoral inédita. Facultad de Ciencias Naturales. La Plata.

JENNINGS, Justin

2005 «La Chichera y el Patrón: Chicha and the Energetics of Feasting in the Prehistoric Andes», en Foundations of Power in the Prehispanic Andes, Kevin Vaugh, Dennis Ogburn y Christina Conlee, eds., pp. 241-259. Archaeological Papers of the AAA vol. 14. Washington DC: Archaeology Division of the American Anthropological Association.

LORENZO, Gabriela

2015 «Análisis zooarqueológico del sitio Cerro Colorado de la Ciénaga de Abajo: una primera aproximación al conjunto arqueofaunístico», en Libro de resúmenes de las X Jornadas de Jóvenes Investigadores en Ciencias Antropológicas, pp. 48. Buenos Aires: INAPL. 
Miante Alzogaray, Ana María y Julián Cámara Hernández

1996 «Restos arqueológicos de maíz (Zea mays ssp. Mays) de Pampa Grande, provincial de Salta, Argentina». Relaciones de la Sociedad Argentina de Antropología 21: 149-159.

MOORE, Jerry

1989 «Pre-Historic Beer in Coastal Peru: Technology and Social Context in Prehistoric Production». American Anthropologist 91 (3): 682-695.

NichOLSON, Edward

1960 «Chicha Maize Types and Chicha Manufacture in Peru». Economic Botany 14 (4): 290-299.

\section{OLISZEWSKI, Nurit}

2004 «Estado actual de las investigaciones arqueobotánicas en sociedades agroalfareras del área valliserrana del NOA (0-600 D.C.)». Relaciones de la Sociedad Argentina de Antropología 29: 211-227.

2005 «Archaeobotany of archaeological sites from Northwest Argentina (1750-1450 B.P.): ceremonial use or rubbish dumps?». International Work Group for Paleoethnobotany 14 (4): 465-471.

2008 «Metodología para la identificación subespecífica de maíces arqueológicos. Un caso de aplicación en el noroeste de argentina», en Arqueobotánica y Teoría Arqueológica, Sonia Archila, Marco Giovannetti y Verónica Lema, eds., pp. 181202. Bogotá: Uniandes-Ceso.

2009 «El recurso maíz en sitios arqueológicos del noroeste argentino: el caso de la Quebrada de los Corrales, el Infiernillo, Tucumán». Treballs d'Etnoarqueologia 7: 83-96.

2012 «La variabilidad racial del maíz y los cambios sociales durante el 1er y $2^{\circ}$ milenio d.C. en el noroeste argentino», en Las manos en la masa, Pilar Babot, María Marschoff y Francisco Pazzarelli, eds., pp. 271-298. Córdoba: Museo de Antropología /CONICET.

OLISZEWSKI, Nurit y Daniel OLIVERA

2009 «Variabilidad racial de macrorrestos arqueológicos de Zea mays (poaceae) y sus relaciones con el proceso agropastoril en la Puna meridional argentina (Antofagasta de la Sierra, Catamarca)». Darwiniana 47 (1): 76-91.

PARODI, Lorenzo

1959 Enciclopedia Argentina de Agricultura y Jardinería 1. Buenos Aires: ACME.

Pochettino, María Lelia y Cristina Scattolin

1991 «Identificación y significado de frutos y semillas carbonizados de sitios arqueológicos formativos de la ladera occidental del Aconquija (Catamarca, República Argentina)». Revista del Museo de La Plata 9 (71): 169-181.

Ramos, Rita, Norma Hilgert y Daniela LAMbaré

2013 «Agricultura tradicional y riqueza de maíces (Zea mays). Estudio de Caso en Caspalá, provincia de Jujuy, Argentina». Boletín de la Sociedad Argentina de Botánica 48 (3-4): 607-621.

Rodríguez, María Fernanda y Carlos Aschero

2007 «Archaeological evidence of Zea mays L. (Poaceae) in the Southern Argentinean Puna (Antofagasta de La Sierra, Catamarca)». Journal of Ethnobiology 27 (2): 256-271. 
SEMPÉ, Carlota

1999 «La Cultura Belén». Actas del XII Congreso Nacional de Arqueología Argentina, tomo II, pp. 250-258. La Plata.

Sempé, Carlota y Mercedes Pérez Meroni

1988 «Nuevo fechado para la cultura Belén, Catamarca. Su evaluación». Resúmenes de las ponencias del IX Congreso Nacional de Arqueología Argentina. Facultad de Filosofía y Letras, Universidad de Buenos Aires.

STALLER, John

2010 Maize Cobs and Cultures: History of Zea Mays L. Berlin: Springer.

VAlencia, Celeste, Marcos Fernández y Carolina BARberis

2010 «Evidencias de incendios en el registro arqueológico de La Ciénaga», en Aldeas protegidas, conflicto y abandono. Investigaciones arqueológicas en La Ciénaga, Bárbara Balesta y Nora Zagorodny, eds., pp. 161-199. La Plata: Al Margen.

WEISER, Vladimiro

1926 Diarios de viaje. Colección Muñiz Barreto alojada en el Departamento Arqueología del Museo de La Plata.

WYNVELDT, Federico

2009 La Loma de los Antiguos de Azampay. Un sitio defensivo del Valle de Hualfín (Catamarca, Argentina). Buenos Aires: Sociedad Argentina de Antropología.

WyNVELDT, Federico y Manuel LóPEZ MATEO

2010 «Pueblos protegidos, murallas y divisaderos: un paisaje arqueológico defensivo en La Ciénaga», en Aldeas protegidas, conflicto y abandono. Investigaciones arqueológicas en La Ciénaga, Bárbara Balesta y Nora Zagorodny, eds., pp. 277-312. La Plata: Al Margen. 